\title{
COMPLETE MAXIMAL SPACELIKE SUBMANIFOLDS
}

\author{
QING-Ming CHENG* AND SUSUMU ISHIKAWA
}

\begin{abstract}
We generalize Simons' method to spacelike submanifolds of $M_{q}^{n+p}(c)$ $(1 \leqq q \leqq p)$ and characterize the totally geodesic submanifolds of $S_{q}^{n+p}(c)(1 \leqq$ $q \leqq p$ ) under the pinching conditions on scalar curvature, Ricci curvature and sectional curvature, respectively.
\end{abstract}

\section{Introduction}

Let $M_{q}^{n+p}(c)$ be an $(n+p)$-dimensional connected indefinite Riemannian manifold of index $q(1 \leqq q \leqq p)$ and of constant curvature $c$, which is called an indefiite space form of index $q$. According to $c>0, c=0$ and $c<0$, it is denoted by $S_{q}^{n+p}(c), \boldsymbol{R}_{q}^{n+p}$ or $H_{q}^{n+p}(c)$. A submanifold $M^{n}$ of an indefinite space form $M_{q}^{n+p}(c)$ is said to be spacelike if the induced metric on $M^{n}$ from that of $M_{q}^{n+p}(c)$ is positive definite. $\boldsymbol{R}^{n}$ can be embedded in $S_{1}^{n+1}(c)$ as a complete totally umbilical spacelike submanifold. But it can not be embedded in the unit sphere $S^{m}(c)$ as a totally umbilical submanifold. Hence it is very interesting to investigate complete spacelike submanifolds in $M_{q}^{n+p}(c)$.

When $p=q$, we know that complete maximal spacelike submanifolds in $S_{p}^{n+p}(\boldsymbol{c})$ or $\boldsymbol{R}_{p}^{n+p}$ are totally geodesic (cf. [3]). Hence the class of all such submanifolds are very small. But if $q<p$ we shall see that the class of complete maximal spacelike submanifolds is very large. In fact, if $M^{n}$ is a complete minimal submanifold in sphere $S^{m}(c)(m>n)$ of constant curvature $c$ embeded in $S_{q}^{m+q}(c)$ as a totally geodesic spacelike submanifold where $m-n+q=p$, then $M^{n}$ is a complete maximal spacelike submanifold in $S_{q}^{n+p}(c)$. In [1], Alias and Romero studied the compact maximal spacelike submanifolds in $S_{q}^{n+p}(c)$. They proved that if $M^{n}$ is a compact maximal spacelike submanifold in $S_{q}^{n+p}(c)$ with Ricci curvature $\operatorname{Ric}\left(M^{n}\right) \geqq(n-1) c$, then $M^{n}$ is totally geodesic. And they indicated that to get a Bernstein type result, the bound on the Ricci curvature

Key words and phrases. Spacelike maximal submanifold, totally geodesic submanifold, scalar curvature, Ricci curvature and sectional curvature.

* Research partially supported by NNSFC, FECC and CPSF

1991 Mathematics Subject Classification 53C20, 53C42

Received September 9, 1996 ; revised May 30, 1997. 
is best possible. But their statement can not guarantee this fact. In fact, according to the theory of minimal submanifold in sphere, we know that there are no $n$-dimensional compact minimal submanifold in $S^{m}(c)$ of which the Ricci curvature satisfies $(n-2) c<\operatorname{Ric}\left(M^{n}\right)<(n-1) c$. Hence the set of examples which they supposed is empty if $(n-2) c<\operatorname{Ric}\left(M^{n}\right)<(n-1) c$.

The purpose of this paper is to generalize the Simons' method to complete spacelike submanifolds in $M_{q}^{n+p}(c)$ and to get the following theorems. In particular, we obtain the best possible bound on the Ricci curvature of a complete maximal spacelike submanifold in the de Sitter space $S_{1}^{n+2}(c)$.

THEOREM 1. Let $M^{n}$ be an $n$-dimensional compact maximal spacelike submanifold in the de Sitter space $S_{q}^{n+p}(c)(1 \leqq q \leqq p)$. If

$$
S \leqq \max \left\{\frac{n c}{2-(1 /(p-q))}, \frac{2 n c}{3}\right\},
$$

then

(1) $M^{n}$ is the totally geodesic submanifold in $S_{q}^{n+p}(c)$, or

(2) $p-q=1, M^{n}$ lies in the totally geodesic spacelike submanifold $S^{n+1}(c)$ of $S_{q}^{n+q+1}(c)$ and is isometric to the Clifford torus $S^{k}((n / k) c) \times S^{n-k}((n /(n-k)) c)$ or

(3) $n=2$ and $p-q=2, M^{2}$ lies in the totally geodesic spacelike submanifold $S^{4}(c)$ of $S_{q}^{4+q}(c)$ and is isometric to the Veronese surface where $S$ is the squared norm of the second fundamental form of $M^{n}$.

Remark 1. When $M^{n}$ is an $n$-dimensional complete maximal spacelike submanifold in the de Sitter space $S_{q}^{n+p}(c)(1 \leqq q \leqq p)$, and $S$ satisfies the condition

$$
\sup S<\max \left\{\frac{n c}{2-(1 /(p-q))}, \frac{2 n c}{3}\right\}
$$

we can prove that $M^{n}$ is the totally geodesic submanifold in $S_{q}^{n+p}(c)$.

THEOREM 2. Let $M^{n}$ be an $n$-dimensional compact maximal spacelike submanifold in the de Sitter space $S_{q}^{n+q+1}(c)$. If the sectional curvature $K$ of $M^{n}$ is positive, then $M^{n}$ is the totally geodesic submanifold in $S_{q}^{n+q+1}(c)$.

Remark 2. The Clifford torus $S^{n-k}((n /(n-k)) c) \times S^{k}((n / k) c)$ in $S^{n+1}(c)$ can be embedded in $S_{q}^{n+q+1}(c)$ as a compact maximal spacelike submanifold with nonnegative curvature and it is not totally geodesic. Hence, the bound on the sectional curvature is best possible.

THEOREM 3. Let $M^{n}$ be an $n$-dimensional complete maximal spacelike submanifold in the de Sitter space $S_{1}^{n+2}(c)$. If $\operatorname{Ric}\left(M^{n}\right) \geqq(n-2) c$, then $M^{n}$ is totally geodesic submanifold in $S_{1}^{n+2}(c)$ or $M^{n}$ is a maximal spacelike Einstein submanifold with $\operatorname{Ric}\left(M^{n}\right)=(n-2) c$ and the parallel second fundamental form.

Remark 3. Let $n=2 k$. The Clifford torus $S^{k}(2 c) \times S^{k}(2 c)$ of $S^{n+1}(c)$ can be 
embedded in $S_{1}^{n+2}(c)$ as a compact spacelike maximal submanifold with $\operatorname{Ric}\left(M^{n}\right)$ $=(n-2) c$ and the parallel second fundamental form. It is open for authors whether there exist the other compact maximal spacelike submanifolds in $S_{1}^{n+2}(c)$ with $\operatorname{Ric}\left(M^{n}\right)=(n-2) c$, and the parallel second fundamental form except the Clifford torus $S^{k}(2 c) \times S^{k}(2 c)$.

Acknowledgement. Authors would like to express here their gratitude to the referee for his valuable suggestions.

\section{Preliminaries}

Let $M_{q}^{n+p}(c)$ be an $(n+p)$-dimensional connected indefinite space form of constant curvature $c$ whose index is $q(1 \leqq q \leqq p)$ and $M^{n}$ an $n$-dimensional connected Riemannian manifold immersed in $M_{q}^{n+p}(c)$. We choose a local frame of orthonormal vector fields $\left\{e_{1}, \ldots, e_{n+p}\right\}$ adapted to the indefinite Riemannian metric of $M_{q}^{n+p}(c)$ and the dual coframe $\left\{\omega_{1}, \ldots, \omega_{n+p}\right\}$ in such a way that, restricted to the submanifold $M^{n},\left\{e_{1}, \ldots, e_{n}\right\}$ are tangent to $M^{n}$. Then the connection forms $\left\{\omega_{A B}\right\}$ of $M_{q}^{n+p}(c)$ are characterized by the structure equations

$$
\left\{\begin{array}{c}
d \omega_{A}=-\sum_{B=1}^{n+p} \varepsilon_{B} \omega_{A B} \wedge \omega_{B}, \quad \omega_{A B}+\omega_{B A}=0 \\
d \omega_{A B}=-\sum_{C=1}^{n+p} \varepsilon_{C} \omega_{A C} \wedge \omega_{C B}-\frac{1}{2} \sum_{C, D=1}^{n+p} \varepsilon_{C} \varepsilon_{D} K_{A B C D} \omega_{C} \wedge \omega_{D}, \\
K_{A B C D}=c \varepsilon_{A} \varepsilon_{B}\left(\delta_{A D} \delta_{B C}-\delta_{A C} \delta_{B D}\right),
\end{array}\right.
$$

where $\varepsilon_{A}=1$ for $1 \leqq A \leqq n+p-q, \varepsilon_{A}=-1$ for $n+p-q+1 \leqq A \leqq n+p$ and $K_{A B C D}$ denotes the components of indefinite Riemannian curvature tensor of $M_{q}^{n+p}(c)$.

The canonic forms $\left\{\omega_{A}\right\}$ and connection forms $\left\{\omega_{A B}\right\}$ restricted to $M^{n}$ are also denoted by the same symbols. We then see

$$
\omega_{\alpha}=0, \quad \alpha=n+1, \ldots, n+p,
$$

and $\left\{e_{1}, \ldots, e_{n}\right\}$ is a local frame of orthonormal vector fields adapted to the in duced Riemannian metric on $M^{n}$ and $\left\{\omega_{1}, \ldots, \omega_{n}\right\}$ is its dual coframe on $M^{n}$. It follows from (2.1), (2.2) and Cartan's Lemma that

$$
\omega_{\alpha \imath}=\sum_{j=1}^{n} h_{i j}^{\alpha} \omega_{j}, \quad h_{\imath \jmath}^{\alpha}=h_{j i}^{\alpha} .
$$

The second fundamental form $\Pi$ and the mean curvature vector $\boldsymbol{h}$ of $M^{n}$ are defined by

$$
\Pi=\sum_{\alpha=n+1}^{n+p} \sum_{\imath, j=1}^{n} \varepsilon_{\alpha} h_{i j}^{\alpha} \omega_{i} \omega_{j} e_{\alpha}
$$

and 


$$
\boldsymbol{h}=\frac{1}{n} \sum_{\alpha=n+1}^{n+p} \varepsilon_{\alpha}\left(\sum_{\imath=1}^{n} h_{i i}^{\alpha}\right) e_{\alpha}
$$

respectively. The mean curvature $H$ of $M^{n}$ is defined by

$$
H=\frac{1}{n} \sqrt{\sum_{\alpha=n+1}^{n+p}\left(\sum_{i=1}^{n} h_{i i}^{\alpha}\right)^{2}} .
$$

If $H=0$, we recall that $M^{n}$ is maximal. Let

$$
S=\sum_{\alpha=n+1}^{n+p} \sum_{\imath, j=1}^{n}\left(h_{\imath j}^{\alpha}\right)^{2}
$$

denote the squared norm of the second fundamental form $\Pi$ of $M^{n}$. The connection forms of $M^{n}$ are characterized by the structure equations

$$
\begin{gathered}
d \omega_{i}=-\sum_{j=1}^{n} \omega_{i \jmath} \wedge \omega_{j}, \quad \omega_{i j}+\omega_{j i}=0, \\
d \omega_{i \jmath}=-\sum_{k=1}^{n} \omega_{i k} \wedge \omega_{k j}-\frac{1}{2} \sum_{k, l=1}^{n} R_{\imath j k l} \omega_{k} \wedge \omega_{l}
\end{gathered}
$$

where $R_{\imath j k l}$ are the components of the curvature tensor of $M^{n}$, that is,

$$
\begin{aligned}
R_{\imath j k l}= & c\left(\delta_{i l} \delta_{j k}-\delta_{i k} \delta_{j l}\right)+\sum_{\alpha=n+1}^{n+p-q}\left(h_{i l}^{\alpha} h_{j k}^{\alpha}-h_{i k}^{\alpha} h_{j l}^{\alpha}\right) \\
& -\sum_{\alpha=n+p-q+1}^{n+p}\left(h_{i l}^{\alpha} h_{j k}^{\alpha}-h_{i k}^{\alpha} h_{j l}^{\alpha}\right) .
\end{aligned}
$$

Letting $R_{\imath}$ and $r$ denote the components of the Ricci curvature and the scalar curvature of $M^{n}$ respectively, we have from (2.10)

$$
\begin{aligned}
R_{j k}= & (n-1) c \boldsymbol{\delta}_{j k}+\sum_{\alpha=n+1}^{n+p-q}\left(\left(\sum_{i=1}^{n} h_{i i}^{\alpha}\right) h_{j k}^{\alpha}-\sum_{i=1}^{n} h_{i k}^{\alpha} h_{j i}^{\alpha}\right) \\
& -\sum_{\alpha=n+p-q+1}^{n+p}\left(\left(\sum_{i=1}^{n} h_{i i}^{\alpha}\right) h_{j k}^{\alpha}-\sum_{i=1}^{n} h_{i k}^{\alpha} h_{j i}^{\alpha}\right)
\end{aligned}
$$

and

$$
\begin{aligned}
r= & n(n-1) c+\sum_{\alpha=n+1}^{n+p-q}\left(\sum_{i=1}^{n} h_{i i}^{\alpha}\right)^{2}-\sum_{\alpha=n+1}^{n+p-q} \sum_{\imath, j=1}^{n}\left(h_{\imath j}^{\alpha}\right)^{2} \\
& -\sum_{\alpha=n+p-q+1}^{n+p}\left(\sum_{\imath=1}^{n} h_{i i}^{\alpha}\right)^{2}+\sum_{\alpha=n+p-q+1}^{n+p} \sum_{\imath, j=1}^{n}\left(h_{i j}^{\alpha}\right)^{2}
\end{aligned}
$$

respectively. We also have

$$
d \omega_{\alpha \beta}=-\sum_{\gamma=n+1}^{n+p} \varepsilon_{\gamma} \omega_{\alpha \gamma} \wedge \omega_{\gamma \beta}-\frac{1}{2} \sum_{\imath, j=1}^{n} R_{\alpha \beta i j} \omega_{i} \wedge \omega_{j},
$$




$$
R_{\alpha \beta \imath \jmath}=-\sum_{l=1}^{n}\left(h_{i l}^{\alpha} h_{l j}^{\beta}-h_{j l}^{\alpha} h_{l \imath}^{\beta}\right) .
$$

By taking the exterior differentiation of (2.3) and defining $h_{\imath j k}^{\alpha}$ by

$$
\sum_{k=1}^{n} h_{i j k}^{\alpha} \omega_{k}=d h_{i j}^{\alpha}-\sum_{k=1}^{n} h_{i k}^{\alpha} \omega_{k j}-\sum_{k=1}^{n} h_{j k}^{\alpha} \omega_{k i}-\sum_{\beta=n+1}^{n+p} \varepsilon_{\beta} h_{i j}^{\beta} \omega_{\beta \alpha},
$$

we get the Codazzi equation

$$
h_{i j k}^{\alpha}=h_{i k J}^{\alpha}=h_{j i k}^{\alpha} \text {. }
$$

We take the exterior differentiation of $(2.15)$ and define $h_{\imath j k l}^{\alpha}$ by

$$
\begin{aligned}
\sum_{l=1}^{n} h_{l j k l}^{\alpha} \omega_{l}= & d h_{l j k}^{\alpha}-\sum_{l=1}^{n} h_{l j k}^{\alpha} \omega_{l i}-\sum_{l=1}^{n} h_{i l k}^{\alpha} \omega_{l \jmath} \\
& -\sum_{l=1}^{n} h_{i j l}^{\alpha} \omega_{l k}-\sum_{\beta=n+1}^{n+p} \varepsilon_{\beta} h_{i j k}^{\beta_{j} \omega_{\beta \alpha} .}
\end{aligned}
$$

Hence, the Ricci formula for the second fundamental form is given by

$$
h_{\imath j k l}^{\alpha}-h_{\imath j l k}^{\alpha}=-\sum_{m-1}^{n} h_{m j}^{\alpha} R_{m i k l}-\sum_{m=1}^{n} h_{\imath m}^{\alpha} R_{m j k l}-\sum_{\beta=n+1}^{n+p} \varepsilon_{\beta} h_{i j}^{\beta} R_{\beta \alpha k l} .
$$

The Laplacian $\Delta h_{\imath \jmath}^{\alpha}$ of $h_{\imath \jmath}^{\alpha}$ is defined by

$$
\Delta h_{i j}^{\alpha}=\sum_{k=1}^{n} h_{i j k k}^{\alpha} .
$$

From the Codazzi equation (2.16) and the Ricci formula (2.18) we get, for the maximal submanifold $M^{n}$ in $M_{q}^{n+p}(c)$,

$$
\begin{aligned}
\Delta h_{i j}^{\alpha}= & \sum_{k=1}^{n} h_{k i j k}^{\alpha}=\sum_{k=1}^{n} h_{k i k j}^{\alpha}-\sum_{k, m=1}^{n} h_{k m}^{\alpha} R_{m \imath j k}-\sum_{k, m=1}^{n} h_{m i}^{\alpha} R_{m k j k} \\
& -\sum_{k=1}^{n} \sum_{\beta=n+1}^{n+p} \varepsilon_{\beta} h_{k i}^{\beta} R_{\beta \alpha j k} \\
= & -\sum_{k, m=1}^{n} h_{k m}^{\alpha} R_{m \imath j k}-\sum_{k, m=1}^{n} h_{m i}^{\alpha} R_{m k j k}-\sum_{k=1}^{n} \sum_{\beta=n+1}^{n+p} \varepsilon_{\beta} h_{k i}^{\beta} R_{\beta \alpha j k} .
\end{aligned}
$$

Thus we get

LEMMA. For the squared norm $S$ of the second fundamental form of the maximal submanifold $M^{n}$ in $M_{q}^{n+p}(c)$, we have

$$
\begin{aligned}
\frac{1}{2} \Delta S & =\sum_{\alpha=n+1}^{n+p} \sum_{\imath, k=1}^{n}\left(h_{\imath j k}^{\alpha}\right)^{2}+\sum_{\alpha=n+1}^{n+p} \sum_{\imath, \jmath=1}^{n} h_{\imath j}^{\alpha} \Delta h_{\imath \jmath}^{\alpha} \\
& =\sum_{\alpha=n+1}^{n+p} \sum_{\imath, k=1}^{n}\left(h_{\imath j k}^{\alpha}\right)^{2}-\sum_{\alpha=n+1}^{n+p} \sum_{\imath, j, m=1}^{n+p} h_{i j}^{\alpha} h_{k m}^{\alpha} R_{m i j k}
\end{aligned}
$$




$$
-\sum_{\alpha=n+1}^{n+p} \sum_{\imath, \jmath, k, m=1}^{n} h_{i j}^{\alpha} h_{m i}^{\alpha} R_{m k j k}-\sum_{\alpha, \beta=n+1}^{n+p} \sum_{\imath, j, k=1}^{n} \varepsilon_{\beta} h_{i j}^{\alpha} h_{k i}^{\beta} R_{\beta \alpha j k} .
$$

\section{Proofs of theorems}

We define $S_{1}$ and $S_{2}$ by

$$
S_{1}:=\sum_{\alpha=n+1}^{n+p-q} \sum_{\imath, j=1}^{n}\left(h_{\imath j}^{\alpha}\right)^{2}, \quad S_{2}:=\sum_{\alpha=n+p-q+1}^{n+p} \sum_{\imath, j=1}^{n}\left(h_{i j}^{\alpha}\right)^{2},
$$

respectively. Then

$$
S=S_{1}+S_{2}
$$

Proof of Theorem 1. Since

$$
\begin{aligned}
\sum_{\alpha=n+1}^{n+p} \sum_{\imath, j, l=1}^{n} h_{l j}^{\alpha} h_{k l}^{\alpha} R_{l \imath j k}= & -c \sum_{\alpha=n+1}^{n+p} \sum_{\imath, j=1}^{n}\left(h_{\imath j}^{\alpha}\right)^{2} \\
& +\sum_{\alpha, \beta=n+1}^{n+p} \sum_{\imath, j, k, l=1}^{n} \varepsilon_{\beta} h_{\imath j}^{\alpha} h_{k l}^{\alpha}\left(h_{l k}^{\beta} h_{i j}^{\beta}-h_{l j}^{\beta} h_{i k}^{\beta}\right) . \\
\sum_{\alpha=n+1}^{n+p} \sum_{\imath, k, l=1}^{n} h_{l j}^{\alpha} h_{l i}^{\alpha} R_{l k j k}= & c \sum_{\alpha=n+1}^{n+p} \sum_{\imath, j=1}^{n}\left(h_{\imath j}^{\alpha}\right)^{2}-n c \sum_{\alpha=n+1}^{n+p} \sum_{l, j=1}^{n}\left(h_{\imath j}^{\alpha}\right)^{2} \\
& +\sum_{\alpha, \beta=n+1}^{n+p} \sum_{\imath, k}^{n} \varepsilon_{\beta} h_{\imath j}^{\alpha} h_{l i}^{\alpha}\left(h_{l k}^{\beta} h_{k j}^{\beta}-h_{l j}^{\beta} h_{k k}^{\beta}\right)
\end{aligned}
$$

and

$$
\sum_{\alpha, \beta=n+1}^{n+p} \sum_{\imath, j, k=1}^{n} \varepsilon_{\beta} h_{\imath j}^{\alpha} h_{k i}^{\beta} R_{\beta \alpha j k}=-\sum_{\alpha, \beta=n+1}^{n+p} \sum_{\imath, j, l=1}^{n} \varepsilon_{\beta} h_{l j}^{\alpha} h_{k i}^{\beta}\left(h_{l k}^{\alpha} h_{l j}^{\beta}-h_{l j}^{\alpha} h_{l k}^{\beta}\right),
$$

we conclud, by using Lemma in the section 2 ,

$$
\begin{aligned}
\frac{1}{2} \Delta S= & \sum_{\alpha=n+1}^{n+p} \sum_{l, j, k=1}^{n}\left(h_{\imath j k}^{\alpha}\right)^{2}+n c \sum_{\alpha=n+1}^{n+p} \sum_{\imath, j, k, l=1}^{n}\left(h_{\imath j}^{\alpha}\right)^{2} \\
& -\sum_{\alpha, \beta=n+1}^{n+p} \sum_{\imath, j, k, l=1}^{n} \varepsilon_{\beta} h_{l j}^{\alpha} h_{k l}^{\alpha}\left(h_{l k}^{\beta} h_{i j}^{\beta}-h_{l j}^{\beta} h_{i k}^{\beta}\right) \\
& -\sum_{\alpha, \beta=n+1}^{n+p} \sum_{\imath, j, l=1}^{n} \varepsilon_{\beta} h_{\imath j}^{\alpha} h_{l i}^{\alpha}\left(h_{l k}^{\beta} h_{k j}^{\beta}-h_{l j}^{\beta} h_{k k}^{\beta}\right) \\
& +\sum_{\alpha, \beta=n+1}^{n+p} \sum_{l, j, l=1}^{n} \varepsilon_{\beta} h_{i j}^{\alpha} h_{k i}^{\beta}\left(h_{l k}^{\alpha} h_{l j}^{\beta}-h_{l j}^{\alpha} h_{l k}^{\beta}\right) \\
= & \sum_{\alpha=n+1}^{n+p} \sum_{l, j, k=1}^{n}\left(h_{i j k}^{\alpha}\right)^{2}+n c \sum_{\alpha=n+1}^{n+p} \sum_{\imath, j, l=1}^{n}\left(h_{i j}^{\alpha}\right)^{2} \\
& -\sum_{\alpha, \beta=n+1}^{n+p} \varepsilon_{\beta}\left[\operatorname{trace}\left(H_{\alpha} H_{\beta}\right)\right]^{2}-\sum_{\alpha, \beta=n+1}^{n+p} \varepsilon_{\beta} N\left(H_{\alpha} H_{\beta}-H_{\beta} H_{\alpha}\right)
\end{aligned}
$$


where $H_{\alpha}=\left(h_{\imath j}^{\alpha}\right)$. Here we denote $N(A)=\operatorname{trace}\left(A^{t} A\right)$ for the $n \times n$-matrix $A=$ $\left(a_{\imath j}\right)$ and the transposed matrix $A^{t}$ of $A$. Then we know $N\left(H_{\alpha} H_{\beta}-H_{\beta} H_{\alpha}\right) \geqq 0$ for any $\alpha$ and $\beta$. Moreover, we put $S_{\alpha \beta}=\sum_{i, j=1}^{n} h_{i j}^{\alpha} h_{i j}^{\beta}$, then the $(p \times p)$-matrix $\left(S_{\alpha \beta}\right)$ is symmetric. So we can choose $\left\{e_{n+1}, \ldots, e_{n+p}\right\}$ such that $\left(S_{\alpha \beta}\right)$ is diagonal.

Now we divide the proof of Theorem 1 into two cases.

Case 1. $p-q=1$. From (3.1), we have

$$
\begin{aligned}
\frac{1}{2} \Delta S= & \sum_{\alpha=n+1}^{n+p} \sum_{\imath, j, k=1}^{n}\left(h_{i j k}^{\alpha}\right)^{2}+n c \sum_{\alpha=n+1}^{n+p} \sum_{\imath, j=1}^{n}\left(h_{i j}^{\alpha}\right)^{2}+\sum_{\alpha, \beta=n+2}^{n+p} N\left(H_{\alpha} H_{\beta}-H_{\beta} H_{\alpha}\right) \\
& +\sum_{\alpha, \beta=n+2}^{n+p}\left[\operatorname{trace}\left(H_{\alpha} H_{\beta}\right)\right]^{2}-N\left(H_{n+1}\right)^{2} \\
\geqq & n c \sum_{\alpha=n+1}^{n+p} \sum_{i, j=1}^{n}\left(h_{i j}^{\alpha}\right)^{2}-N\left(H_{n+1}\right)^{2}+\sum_{\alpha=n+2}^{n+p} N\left(H_{\alpha}\right)^{2} \\
\geqq & n c S-S^{2} .
\end{aligned}
$$

From the assumptions in Theorem 1 and the Stokes formula, we get $S=0$ or $S=n c$. If $S=0$, then $M^{n}$ is totally geodesic. If $S=n c$, from the above (3.2), we know $S_{2}=0$ on $M^{n}$, i.e., $h_{i j}^{\alpha}=0$ on $M^{n}$ for $\alpha=n+2, \ldots, n+p$. Hence $M^{n}$ lies in the totally geodesic spacelike submanifold $S^{n+1}(c)$ of $S_{q}^{n+q+1}(c)$ (see Theorem 1 in [6]). Thus $M^{n}$ becomes a compact minimal hypersurface in $S^{n+1}(c)$ such that the squared norm $S$ of the second fundamental form is equal to $n c$. From the result due to Chern-do Carmo and Kobayashi [2], we know that $M^{n}$ is isometric to the Clifford torus. We complete the proof of Theorem 1 in this case.

Case 2. $p-q>1$. In this case, we have

$$
\begin{aligned}
\frac{1}{2} \Delta S= & \sum_{\alpha=n+1}^{n+p} \sum_{\imath, j=1}^{n}\left(h_{i j k}^{\alpha}\right)^{2}+n c \sum_{\alpha=n+1}^{n+p} \sum_{\imath, j=1}^{n}\left(h_{i j}^{\alpha}\right)^{2} \\
& +\sum_{\alpha, \beta=n+p-q+1}^{n+p} N\left(H_{\alpha} H_{\beta}-H_{\beta} H_{\alpha}\right)+\sum_{\alpha, \beta=n+p-q+1}^{n+p}\left[\operatorname{trace}\left(H_{\alpha} H_{\beta}\right)\right]^{2} \\
& -\sum_{\alpha, \beta=n+1}^{n+p-q} N\left(H_{\alpha} H_{\beta}-H_{\beta} H_{\alpha}\right)-\sum_{\alpha, \beta=n+1}^{n+p-q}\left[\operatorname{trace}\left(H_{\alpha} H_{\beta}\right)\right]^{2} .
\end{aligned}
$$

From a Lemma due to $\mathrm{Li}-\mathrm{Li}$ in [4], we get

$$
-\sum_{\alpha, \beta=n+1}^{n+p-q} N\left(H_{\alpha} H_{\beta}-H_{\beta} H_{\alpha}\right)-\sum_{\alpha, \beta=n+1}^{n+p-q}\left[\operatorname{trace}\left(H_{\alpha} H_{\beta}\right)\right]^{2} \geqq-\frac{3}{2}\left[\sum_{\alpha=n+1}^{n+p-q} \sum_{2, j=1}^{n}\left(h_{\imath j}^{\alpha}\right)^{2}\right]^{2} .
$$

Hence, we get

$$
\frac{1}{2} \Delta S \geqq\left(n c S-\frac{3}{2} S^{2}\right)+\sum_{\alpha=n+p-q+1}^{n+p} N\left(H_{\alpha}\right)^{2} .
$$

From the Stokes formula, the assumptions in Theorem 1 and (3.3), we get $S=$ $(2 / 3) n c$ or $S=0$. If $S=0$, then $M^{n}$ is totally geodesic. If $S=(2 / 3) n c$, we know 
$h_{\imath \jmath}^{\alpha}=0$ on $M^{n}$ for $\alpha=n+p-q, \ldots, n+p$. Hence $M^{n}$ lies in the totally geodesic spacelike submanifold $S^{n+p-q}(c)$ of $S_{q}^{n+p}(c)$ (see Theorem 1 in [6]). Thus $M^{n}$ becomes a compact minimal submanifold in $S^{n+p-q}(c)$ such that the squared norm $S$ of the second fundamental form is equal to $(2 / 3) n c$. From the result due to $\mathrm{Li}-\mathrm{Li}$ [4], we know that $n=p-q=2$ and $M^{n}$ is isometric to a Veronese surface. Theorem 1 holds in this case. We complete the proof of Theorem 1 .

Proposition. Let $M^{n}$ be an $n$-dimensional compact maximal spacelike submanifold in the de Sitter space $S_{q}^{n+q+1}(c)$. It the sectional curvature $K$ of $M^{n}$ is nonnegative, then $M^{n}$ is totally geodesic or $M^{n}$ is a compact maximal spacelike submanifold with parallel second fundamental form.

Proof of Proposition. For any fixed $\alpha$, we can choose $e_{1}, \ldots, e_{n}$ such that $h_{i j}^{\alpha}=\lambda_{i}^{\alpha} \delta_{i j}$. Then we have

$$
\begin{aligned}
& -\sum_{\imath, \jmath, k, l=1}^{n} h_{\imath j}^{\alpha} h_{k l}^{\alpha} R_{l \imath j k}-\sum_{\imath, \jmath, k, l=1}^{n} h_{\imath j}^{\alpha} h_{l \imath}^{\alpha} R_{l k \jmath k} \\
& =-\sum_{\imath, k=1}^{n} \lambda_{\imath}^{\alpha} \lambda_{k}^{\alpha} R_{k \imath \imath k}-\sum_{\imath, k=1}^{n}\left(\lambda_{\imath}^{\alpha}\right)^{2} R_{\imath k i k} \\
& =\frac{1}{2} \sum_{\imath, k=1}^{n}\left(\lambda_{\imath}^{\alpha}-\lambda_{k}^{\alpha}\right)^{2} R_{k \imath \imath k} \\
& \quad \geqq \frac{1}{2} \sum_{i, k=1}^{n}\left(\lambda_{\imath}^{\alpha}-\lambda_{k}^{\alpha}\right)^{2} K_{0}=n K_{\theta} \sum_{\imath, k=1}^{n}\left(h_{i k}^{\alpha}\right)^{2},
\end{aligned}
$$

where $K_{0}$ denotes the infimum of the sectional curvature of $M^{n}$. Since the both sides of the above inequality do not depend on the choice of the orthonormal frame $\left\{e_{1}, \ldots, e_{n}\right\}$, we have

$$
\begin{aligned}
& -\sum_{\alpha=n+1}^{n+q+1} \sum_{\imath, j, l=1}^{n} h_{l j}^{\alpha} h_{k l}^{\alpha} R_{l \imath j k}-\sum_{\alpha=n+1}^{n+q+1} \sum_{k, l=1}^{n} h_{i j}^{\alpha} h_{l i}^{\alpha} R_{l k j k} \\
& \geqq n K_{0} \sum_{\alpha=n+1}^{n+q+1} \sum_{l, j=1}^{n}\left(h_{\imath j}^{\alpha}\right)^{2} \geqq n K_{0} S .
\end{aligned}
$$

From Lemma and (3.4), we get

$$
\begin{aligned}
\frac{1}{2} \Delta S & =\sum_{\alpha=n+1}^{n+q+1} \sum_{\imath, k=1}^{n}\left(h_{\imath j k}^{\alpha}\right)^{2}-\sum_{\alpha=n+1}^{n+q+1} \sum_{\imath, k}^{n} h_{m=1}^{\alpha} h_{i j}^{\alpha} h_{k m}^{\alpha} R_{m \imath j k} \\
& -\sum_{\alpha=n+1}^{n+q+1} \sum_{\imath, j, k, m=1}^{n} h_{i j}^{\alpha} h_{m i}^{\alpha} R_{m k j k}-\sum_{\alpha, \beta=n+1}^{n+q+1} \sum_{\imath, j=1}^{n} \varepsilon_{\beta} h_{\imath j}^{\alpha} h_{k i}^{\beta} R_{\beta \alpha j k} \\
& =\sum_{\alpha=n+1}^{n+q+1} \sum_{\imath, k=1}^{n}\left(h_{i j k}^{\alpha}\right)^{2}-\sum_{\alpha=n+1}^{n+q+1} \sum_{k, m=1}^{n} h_{\imath j}^{\alpha} h_{k m}^{\alpha} R_{m \imath j k} \\
& -\sum_{\alpha=n+1}^{n+q+1} \sum_{\imath, j, k}^{n} h_{i j}^{\alpha} h_{m i}^{\alpha} R_{m k j k}-\frac{1}{2} \sum_{\alpha, \beta=n+1}^{n+q+1} \varepsilon_{\beta} N\left(H_{\alpha} H_{\beta}-H_{\beta} H_{\alpha}\right)
\end{aligned}
$$




$$
\begin{aligned}
& \geqq n K_{0} S+\frac{1}{2} \sum_{\alpha, \beta=n+2}^{n+q+1} N\left(H_{\alpha} H_{\beta}-H_{\beta} H_{\alpha}\right) \\
& \geqq n K_{0} S .
\end{aligned}
$$

Since the sectional curvature of $M^{n}$ is nonnegative, we have $K_{0} \geqq 0$. Hence, from the Stokes formula, we obtain $S=0$, i.e., $M^{n}$ is totally geodesic or $S$ is constant and $h_{i j_{k}}^{\alpha}=0$. We complete the proof of Proposition.

Proof of Theorem 2. From Proposition and its proof, it is obvious that Theorem 2 holds.

Proof of Theorem 3. From the assumptions of Theorem 3 and Myers Theorem, we know that $M^{n}$ is compact. According to (3.1), we get

$$
\begin{aligned}
\frac{1}{2} \Delta S & =\sum_{\alpha=n+1}^{n+2} \sum_{\imath, k=1}^{n}\left(h_{i j k}^{\alpha}\right)^{2}+n c \sum_{\alpha=n+1}^{n+2} \sum_{\imath, j=1}^{n}\left(h_{i j}^{\alpha}\right)^{2} \\
& -\sum_{\alpha, \beta=n+1}^{n+2} \varepsilon_{\beta} N\left(H_{\alpha} H_{\beta}-H_{\beta} H_{\alpha}\right)-\sum_{\alpha, \beta=n+1}^{n+2} \varepsilon_{\beta}\left[\operatorname{trace}\left(H_{\alpha} H_{\beta}\right)\right]^{2} \\
& \geqq n c \sum_{\alpha=n+1}^{n+2} \sum_{i, j=1}^{n}\left(h_{i j}^{\alpha}\right)^{2}-N\left(H_{n+1}\right)^{2}+N\left(H_{n+2}\right)^{2} .
\end{aligned}
$$

Hence we have

$$
\frac{1}{2} \Delta S \geqq\left(n c-S_{1}+S_{2}\right) S
$$

where $S_{1}=N\left(H_{n+1}\right), S_{2}=N\left(H_{n+2}\right)$ and $S=S_{1}+S_{2}$. By using (2.11) and the assumption $\operatorname{Ric}\left(M^{n}\right) \geqq(n-2) c$ in Theorem 3 , we have

$$
c-\sum_{i=1}^{n}\left(h_{i j}^{n+1}\right)^{2}+\sum_{i=1}^{n}\left(h_{i j}^{n+2}\right)^{2} \geqq 0 .
$$

Thus

$$
n c-S_{1}+S_{2} \geqq 0 .
$$

From (3.5) and (3.6), we conclude

$$
\sum_{\alpha=n+1}^{n+2} \sum_{\imath, j=1}^{n}\left(h_{\imath j k}^{\alpha}\right)^{2}=0
$$

and $S=0$ or $n c-S_{1}+S_{2}=0$ and $S$ is constant. If $S=0$, then $M^{n}$ is totally geodesic. If $S \neq 0$, then all of the above inequalities become equalities. Hence, the Ricci curvature is equal to $(n-2) c$. We complete the proof of Theorem 3.

\section{REFERENCES}

[1] L. J. Alias AND A. Romero, Integral formulas for compact spacelike $n$-submani- 
folds in de Sitter spaces Applications to the papallel mean curvature vector case, Manuscripta Math., 87 (1995), 405-416.

[2] S.S. Chern, M. do Carmo and S. Kobayashi, Minimal submanifolds of a sphere with second fundamental form of constant length., Functional Analysis and Related Fields, Springer, New York, 1970, pp. 59-75.

[3] T. IsHiHARA, Maximal spacelike submanifolds of a pseudo-Riemannian space of constant curvature., Michigan Math. J., 35 (1988), 345-352.

[4] A.M. LI AND J.M. LI, An intrinsic rigidity theorem for minimal submanifolds in a sphere, Arch. Math., 58 (1992), 582-594.

[5] J. Simons, Minimal varieties in Riemannian manifolds, Ann. of Math., 88 (1968), 62-105.

[6] S. T. YAU, Submanifolds with constant mean curvature I, Amer. J. Math., 96 (1974), 346-366.

Department of Mathematics

FACULTY OF SCIENCE

JOSAI UNIVERSITY

SAKado, SAITAMA 350-02

JAPAN

Department of Mathematics

SAGa UNIVERSITY

SAGA 840

JAPAN 